\title{
Specificity of esthetic experience for artworks: an fMRI study
}

\author{
Cinzia Di Dio ${ }^{1}$, Nicola Canessa ${ }^{2}$, Stefano F. Cappa ${ }^{2}$ and Giacomo Rizzolatti ${ }^{1,3}$ * \\ ' Department of Neuroscience, Università degli Studi di Parma, Parma, Italy \\ ${ }^{2}$ Center for Cognitive Neuroscience and CERMAC, Vita-Salute San Raffaele University, Milan, Italy \\ ${ }^{3}$ Brain Center for Social and Motor Cognition, Italian Institute of Technology, Parma, Italy
}

Edited by:

Idan Segev, The Hebrew University of Jerusalem, Israel

\section{Reviewed by:}

Bernd Weber,

Rheinische-Friedrich-Wilhelms

Universität, Germany

Philip D. Zelazo, University of

Minnesota, USA

Son Preminger, Interdisciplinary

Center Herzliya, Israel

\section{*Correspondence:}

Giacomo Rizzolatti, Department of

Neuroscience, Università degli Studi

di Parma, Via Volturno 39/E, 43100

Parma, Italy.

e-mail: giacomo.rizzolatti@unipr.it
In a previous functional magnetic resonance imaging (fMRI) study, where we investigated the neural correlates of esthetic experience, we found that observing canonical sculptures, relative to sculptures whose proportions had been modified, produced the activation of a network that included the lateral occipital gyrus, precuneus, prefrontal areas, and, most interestingly, the right anterior insula. We interpreted this latter activation as the neural signature underpinning hedonic response during esthetic experience. With the aim of exploring whether this specific hedonic response is also present during the observation of non-art biological stimuli, in the present fMRI study we compared the activations associated with viewing masterpieces of classical sculpture with those produced by the observation of pictures of young athletes. The two stimulus-categories were matched on various factors, including body postures, proportion, and expressed dynamism. The stimuli were presented in two conditions: observation and esthetic judgment. The two stimuluscategories produced a rather similar global activation pattern. Direct comparisons between sculpture and real-body images revealed, however, relevant differences, among which the activation of right antero-dorsal insula during sculptures viewing only. Along with our previous data, this finding suggests that the hedonic state associated with activation of right dorsal anterior insula underpins esthetic experience for artworks.

Keywords: neuroesthetics, sculpture, human body, insula

\section{INTRODUCTION}

Neuroesthetics is the field of cognitive neuroscience that investigates the neural bases of esthetic experience. In visual art, esthetic experience appears to be based on an initial visual encoding of the observed artwork (Kawabata and Zeki, 2004) and on subsequent processing carried out in a series of higher order cortical areas (e.g., Vartanian and Goel, 2004; Lacey et al., 2011). Spatial coding (e.g., Cela-Conde et al., 2009; Cupchik et al., 2009), motor activation (Jacobsen et al., 2006; Freedberg and Gallese, 2007), and activation of emotional centers (Jacobsen et al., 2006; Di Dio et al., 2007; Cupchik et al., 2009) are some of the processes that appear to take place during esthetic experience (for a review, see Di Dio and Gallese, 2009).

In a previous study we investigated the neural correlates of esthetic experience during the observation of masterpieces of classical and renaissance sculpture (Di Dio et al., 2007) In this study, sculpture images were presented to participants in two versions: original ("canonical") and proportion-modified. The rationale underlying proportion modification was that, in these masterpieces, proportion is strictly related to esthetic evaluation of the stimuli. By altering proportion in a controlled fashion and by keeping every other factor constant, it was then possible to unfold the neural correlates associated with esthetic experience for these artworks. Furthermore, in this study participants viewed the stimuli in three conditions: observation, esthetic judgment, and proportion judgment. The distinctive feature of this protocol was to allow participants, during observation condition, to observe the images without expressing any explicit judgment. In fact, explicit judgments that require decision-making may induce specific task-related processes that could diminish the neural activation responsible of hedonic responses.

The results showed that, on the whole, independent of stimulus and condition types, the observation of images of classical and renaissance sculptures elicited activation of several visual areas, the inferior parietal lobule (IPL), the ventral premotor cortex plus the adjacent posterior portion of right inferior frontal gyrus (IFG), as well as deep structures, including the hippocampus and the insula.

Most interestingly, the contrast canonical vs. modified sculpture images revealed activation of a brain network, which included cortical areas encoding the physical properties of the stimuli, areas encoding implied motion, and the right anterior insula. The emotional response, hallmarked by insula activation (Mesulam and Mufson, 1982, 1985; Augustine, 1996; Damasio, 1999; Damasio et al., 2000; Craig, 2003; Dupont et al., 2003; Critchley et al., 2004, 2005) was particularly strong during observation condition, in which the participants could be said to respond most spontaneously to the presented images.

Support for the finding that the hedonic dimension of esthetic experience is related to insular activation also comes from a recent study by Cupchik et al. (2009). In this functional magnetic resonance imaging (fMRI) study, participants viewed various categories of paintings (portraits, nudes, still-life, and landscapes) that 
were presented in two conditions: one that required the participants to observe the images in an objective and detached manner to gather information about the content of the stimulus ("pragmatic condition"), and one that required them to observe the paintings in a subjective and engaged manner, appreciating the feelings evoked by the stimuli ("esthetic condition"). Note that, similarly to our "observation" condition, instructions given to the participants prior "esthetic" condition were to experience the mood evoked by the artworks without making any explicit judgment about the stimuli. Results showed that observation of paintings under the "esthetic" condition vs. baseline condition (viewing of paintings accompanied by no explicit task-related instructions) elicited bilateral activation of the insula, suggesting that this area is crucially implicated in the hedonic feeling associated with esthetic experience.

In the present study we investigated, using fMRI, whether the hedonic response associated with esthetic experience when viewing art masterpieces occurs also during the observation of non-art biological stimuli or whether it is distinctive of esthetic experience for artworks. For this purpose, we compared the activations evoked by sculpture images with those produced by the observation of real human body (HB) images depicting young athletes. The athletes posed with body postures that resembled those portrayed in the sculpture images (for details, see Material and Methods). In order to match the body configurations across stimulus-categories, all stimuli represented male figures (see Figure 1 for an example of stimuli).

This study was composed of two experiments. In both of them, we presented the two stimulus-categories (art vs. biological nonart) in two conditions: observation and explicit esthetic judgment. The main difference between the two experiments laid in the stimulus presentation protocol and in the instruction provided to participants prior scanning. In Experiment 1, the stimuli (sculptures and real $\mathrm{HB}$ images) were presented intermixed in a semirandomized order within the same functional runs. This protocol emphasized the differences between the two stimulus-categories. In Experiment 2, each stimulus-category was presented separately in different functional runs. By keeping the two stimuluscategories in separate runs we intended to highlight differences in brain activations distinctive of each stimulus-category.

The results showed a similar, yet not identical, activation pattern for the two stimulus-categories. The direct comparisons between sculpture and real $\mathrm{HB}$ images revealed differences at the visual and, most importantly, at the emotional level of processing. We argue that the activation pattern observed for sculptures images, inclusive of insula activation, pinpoints the hedonic aspect of esthetic experience. This type of experience is lacking when viewing non-art biological stimuli.

\section{MATERIALS AND METHODS PARTICIPANTS}

Thirty-two healthy right-handed Italian students [16 females (mean age $=21.4, \mathrm{SD}=1.23$, range $=19-25$ ) and 16 males (mean age $=23.43, \mathrm{SD}=1.39$, range $=21-30)$ ] participated in Experiment 1 . Twenty-four healthy right-handed Italian students [12 females (mean age $=20.28, \mathrm{SD}=1.16$, range $=19-23$ ) and 12 males (mean age $=22.86, \mathrm{SD}=3.26$, range $=19-30)]$ participated
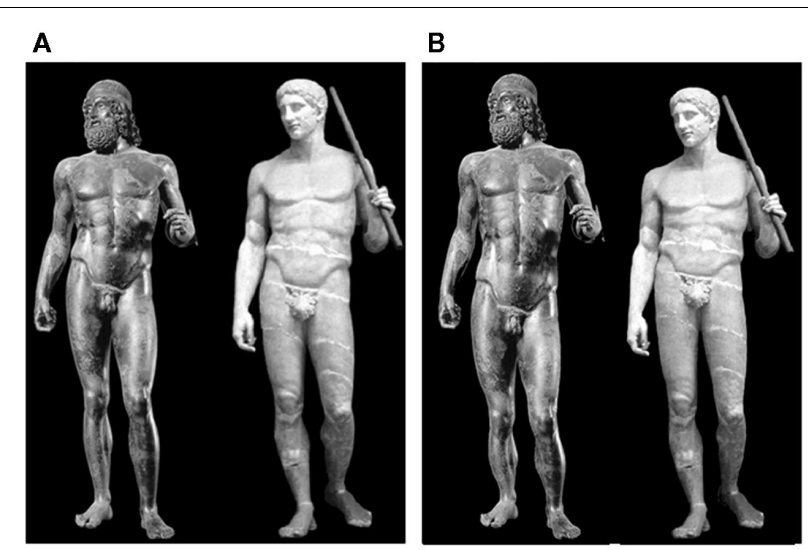

c

D
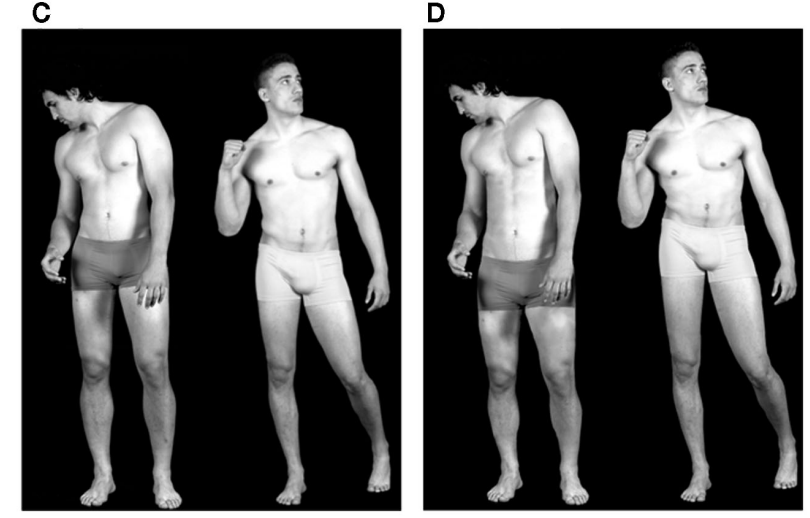

FIGURE 1 | Example of experimental stimuli used in this study. (A) Images of canonical sculptures; (B) images of proportion-modified sculptures; (C) images of canonical real human bodies; (D) images of proportion-modified real human bodies. Proportion-modified images (B,D) are presented with a long trunk-short legs relation (images on the left) and with a short trunk-long legs relation (images on the right).

in Experiment 2. All participants were naïve to art criticism, as assessed during recruitment. They had normal or corrected-tonormal visual acuity. None reported a history of psychiatric or neurological disorders, or current use of any psychoactive medications. They gave their written informed consent to the experimental procedure, which was approved by the Ethics Committee of San Raffaele Scientific Institute (Milan) and Local Ethics Committee of Parma.

\section{STIMULI}

Sixteen two-dimensional images of male sculptures (S) and 16 images of real male human bodies (HB) were chosen following the selection method described in Di Dio et al. (2007). For the present study, stimuli were selected out of an initial pool of images composed of a total 56 images of sculptures (28 canonical and 28 modified - see below) and 56 real HB images ( 28 canonical and 28 modified - see below). In this preliminary behavioral study, which was aimed at stimulus selection for the fMRI experiment, we examined the relation between esthetic judgment and proportion in 22 observers naïve to art criticism. Participants of the behavioral study underwent observation, esthetic judgment and proportion judgment conditions. To assess the probability that the stimuli 
were perceived either proportioned or disproportioned according to our prior categorization (canonical and modified), during proportion judgment, participants had to rate stimuli proportion on a dichotomous measure ( 0 - disproportioned; 1 - proportioned). During esthetic judgment condition, on the other hand, participants had to rate the stimuli on a scale from 0 (ugly) to 7 (beautiful). By using a continuous scale, we aimed at increasing sensitivity on the assessment of the esthetic response to the stimuli, which still needed to be quantified in this preliminary behavioral stage.

The original canonical images of sculptures were chosen from classical examples that met the golden ratio criteria (proportion torso: legs $(\mathrm{T}-\mathrm{L})=0.62 \pm 0.01)$. The real-body images were selected from pictures taken specifically for this study by a professional photographer using athletes, whose body proportion and figure resembled those portrayed in the sculpture images. Also the proportion associated with the selected real-body images met the golden ratio criteria (proportion torso: legs $(\mathrm{T}-\mathrm{L})=0.62 \pm 0.02$ ). Athletes were required to pose following the postures depicted in the sculpture images. All images were black and white and represented only male bodies that were comparable across categories in terms of body structure, proportion between body parts, posture, expressed dynamism. Expressed dynamism of the canonical sculpture and real-body stimuli was assessed by nine independent judges during the preliminary behavioral study aimed at stimulus selection (see above). The criteria according to which the evaluators assigned the stimuli to each category were the following: sense of balance, position of the limbs, feeling of motion, direction of eye-gaze, and facial expression. Based on the judges' rating, stimuli were initially categorized into 10 dynamic and 18 static sculpture images and 12 dynamic and 16 static realbody images. With respect to this further sub-categorization, the stimuli selected for the fMRI study contained an even number of judged-dynamic (8) and judged-static (8) images within each category.

A modified version of sculpture and real-body images was created by altering the proportion between torso and legs (T-L) of the original images, thus producing two new sets of stimuli identical to the formers except for proportion. Using the algorithm employed in the previous experiment (Di Dio et al., 2007), half of the images were modified by shortening the torso and elongating the legs (modification range $\mathrm{T}-\mathrm{L}=0.5-0.6$ ), whereas the other half followed the opposite modification pattern, with long torso and short legs (modification range $\mathrm{T}-\mathrm{L}=0.64-0.75$ ).

An example of the two stimulus-categories (original and modified) is in Figure 1.

\section{PARADIGM AND TASK EXPERIMENT 1}

The stimuli were presented in a $2 \times 2$ design, with two levels of stimulus-category [sculpture (S) and real $\mathrm{HB}(\mathrm{HB})$ ] and two levels of stimulus-type [canonical (C) and modified (M)]. The stimuli were presented in two separate experimental conditions [observation $(\mathrm{O})$ and esthetic judgment $(\mathrm{AJ})$ ]. Each participant underwent eight separate fMRI runs, repeating each experimental condition twice. The condition order was maintained fixed across all participants, with observation condition first (runs 1-4), and explicit esthetic judgment last (runs 5-8). By keeping the observation runs first, we aimed at measuring unbiased (spontaneous) brain responses to the stimuli. The participants expressed their explicit esthetic judgment during the esthetic judgment condition.

Every run comprised 32 trials. Sculpture images were presented in 16 trials, and real HB images were presented in the other 16 . Within each category-specific trials, half of the images (eight) were presented in the canonical version and half (eight) in the modified version. To reduce possible cross-category carry-over cognitive effects, stimuli were presented in a semi-randomized order, with mini-blocks consisting of eight consecutive images of the same stimulus-category (either $\mathrm{S}$ or $\mathrm{HB}$ ), never repeating the same image within a run.

Participants lay in the scanner in a dimly lit environment. The stimuli were viewed via a back-projection screen located in front of the scanner and a mirror placed on the head-coil. The software Presentation 11.0 (Neurobehavioral systems, Albany, CA, USA ${ }^{1}$ ) was used both for stimuli presentation and for the recording of the participants' answers. At the beginning of each run, a $4 \mathrm{~s}$ visual instruction informed the participants about the upcoming condition. On each trial, the stimulus appeared at the center of the screen for $2.5 \mathrm{~s}$ and was followed by a $3 \mathrm{~s}$ blank-screen interval. Subsequently, a question mark instructed the participants to respond to the stimulus in accordance with the task introduced (see below). The question mark remained on screen for $400 \mathrm{~ms}$ and was followed by an inter-stimulus interval (ISI; white-cross fixation) whose duration was varied ("jittered") at every trial, in order to desynchronize the timings of event-types with respect to the acquisition of single slices within functional volumes and to optimize statistical efficiency (Dale, 1999). The OptSeq2 Toolbox $^{2}$ was used to estimate the optimal ISIs (mean ISI $=3.87 \mathrm{~s}$, range $=1.5-19.750 \mathrm{~s}$ ). Each scanning run lasted approximately $6.5 \mathrm{~min}$.

During observation condition $(\mathrm{O})$, the participants were required to simply observe the images and, when the question mark appeared, they had to indicate whether they paid attention to the image or not. During the esthetic judgment condition, they were required to decide whether they esthetically liked the image or not. Thus, both conditions required a response from the participants. Using the index or middle finger of the right hand, the participants answered yes or no, according to the instruction presented at the start of each run. The question "did you pay attention to the image?" was introduced to make sure that participants were actually looking at the stimuli during fMRI scanning.

\section{PARADIGM EXPERIMENT 2}

Participants lay in the scanner in a dimly lit environment. The stimuli were viewed via digital visors (VisuaSTIM) with a 500,000 pixel $\times 0.25$ square inch resolution and horizontal eye field of $30^{\circ}$. The visors were applied directly on the volunteers' face. The digital transmission of the signal to the scanner was via optic fiber. The software E-Prime 2 Professional (Psychology Software Tools, Inc., Pittsburgh, PA, USA ${ }^{3}$ ) was used both for stimulus presentation and recording of the participants' answers.

\footnotetext{
${ }^{1}$ http://www.neurobs.com

2 http://surfer.nmr.mgh.harvard.edu/optseq/

${ }^{3}$ http://www.pstnet.com
} 
The structure of the experimental trials within each run was identical to that described for Experiment 1. Differently from Experiment 1, in Experiment 2 the total duration time of each run doubled (about $12 \mathrm{~min}$ ), making four the total number of functional runs. However, the actual main difference with Experiment 1 laid in how stimuli were presented. In Experiment 1, stimuli presentation was organized in randomized mini-blocks of eight stimuli belonging to the same category (either S or HB). In Experiment 2, instead, half of the participants $(N=13)$ were presented with all sculpture images first (runs 1-2) and then with real $\mathrm{HB}$ images (runs 3-4), and half of the participants were presented with the opposite order. In this way, instructions for each experimental condition (particularly for observation condition, where we aimed at priming the proper mind-state) could be addressed more precisely in accordance with the specific stimulus-category to follow. More specifically, during observation condition of sculpture images the volunteers were required to observe the images as "they were in a museum." During observation condition of real $\mathrm{HB}$, they had to observe images "as if leafing through a magazine where they would have seen boys posing for photograph shots." For both stimulus-categories, participants were instructed to relax and observe the stimuli trying to explore each image in full.

\section{IMRI DATA ACOUISITION}

For Experiment 1, anatomical T1-weighted and functional T2*-weighted MR images were acquired with a $3 \mathrm{~T}$ Philips Achieva scanner (Philips Medical Systems, Best, NL, USA), using an eight-channels Sense head-coil (sense reduction factor $=2$ ). Functional images were acquired using a $\mathrm{T} 2 *$-weighted gradient-echo, echo-planar (EPI) pulse sequence (38 interleaved transverse slices covering the whole brain with the exception of the primary visual cortex and the posterior part of the cerebellum, $\mathrm{TR}=3000 \mathrm{~ms}, \mathrm{TE}=30 \mathrm{~ms}$, flip-angle $=85^{\circ}$, FOV $=240 \mathrm{~mm} \times 240 \mathrm{~mm}$, inter-slice gap $=0.5 \mathrm{~mm}$, slice thickness $=4 \mathrm{~mm}$, in-plane resolution $2.5 \mathrm{~mm} \times 2.5 \mathrm{~mm}$ ). Each scanning sequence comprised 120 sequential volumes. Immediately after the functional scanning a high-resolution T1-weighted anatomical scan ( 150 slices, $\mathrm{TR}=600 \mathrm{~ms}, \mathrm{TE}=20 \mathrm{~ms}$, slice thickness $=1 \mathrm{~mm}$, in-plane resolution $1 \mathrm{~mm} \times 1 \mathrm{~mm}$ ) was acquired for each participant.

For Experiment 2, anatomical T1-weighted and functional T2*weighted MR images were acquired with a $3 \mathrm{~T}$ General Electrics scanner equipped with an eight-channels receiver head-coil. Functional images were acquired using a T2*-weighted gradientecho, EPI pulse sequence (acceleration factor asset 2, 37 interleaved transverse slices covering the whole brain, $\mathrm{TR}=2100 \mathrm{~ms}$, $\mathrm{TE}=30 \mathrm{~ms}$, flip-angle $=90^{\circ}, \mathrm{FOV}=205 \mathrm{~mm} \times 205 \mathrm{~mm}$, interslice gap $=0.5 \mathrm{~mm}$, slice thickness $=3 \mathrm{~mm}$, in-plane resolution $2.5 \mathrm{~mm} \times 2.5 \mathrm{~mm}$ ). Each scanning sequence comprised 306 sequential volumes. Immediately after the functional scanning a high-resolution inversion recovery prepared T1-weighted anatomical scan (acceleration factor arc 2, 156 sagittal slices, matrix $256 \times 256$, isotropic resolution $1 \mathrm{~mm} \times 1 \mathrm{~mm} \times 1 \mathrm{~mm}$, $\mathrm{TI}=450 \mathrm{~ms}, \mathrm{TR}=8100 \mathrm{~ms}, \mathrm{TE}=3.2 \mathrm{~ms}$, flip-angle $12^{\circ}$ ) was acquired for each participant.

\section{fMRI STATISTICAL ANALYSIS}

Image pre-processing and statistical analysis were performed using SPM8 (Wellcome Department of Cognitive Neurology ${ }^{4}$ ), implemented in Matlab v7.6 (Mathworks, Inc., Sherborn, MA, USA; Worsley and Friston, 1995). The first 6 volumes (Experiment 1) and the first four volumes (Experiment 2) of each functional run were discarded to allow for T1 equilibration effects. All remaining volumes from each participant were then spatially realigned (Friston et al., 1996) to the first volume of the first run to correct for between-scan motion, and unwarped (Andersson et al., 2001). A mean-image from the realigned volumes was created. The T1weighted anatomical image was coregistered to such mean-image, and segmented in gray-matter, white matter, and cerebro-spinalfluid. During the segmentation the gray-matter component was automatically normalized to a gray-matter probabilistic map ${ }^{5}$. The derived spatial transformations were then applied to the realignedand-unwarped $\mathrm{T} 2 *$-weighted volumes, that were resampled in $2 \mathrm{~mm} \times 2 \mathrm{~mm} \times 2 \mathrm{~mm}$ voxels after normalization. All functional volumes were then spatially smoothed with an 8 -mm full-width half-maximum (FWHM) isotropic Gaussian kernel to compensate for residual between-subject variability after spatial normalization.

Statistical inference was based on a random-effects approach (Friston et al., 1999). This comprised two steps. At the first (singlesubject) level, fMRI responses were modeled in a design-matrix comprising the onset-times of the following regressors: instruction, stimuli ( $\mathrm{S}$ and $\mathrm{HB}$; C and $\mathrm{M}$ ), blank intervals, and question mark that cued overt responses. Regressors modeling events were convolved with a canonical hemodynamic response function (HRF), and parameter estimates for all regressors were obtained at each voxel by maximum-likelihood estimation. Linear contrasts were used to determine (a) common effects (sculpture vs. baseline and real HB images vs. baseline, for both canonical and modified image types within each stimulus-category), and (b) differential effects associated with the presentation of the sculptures (C-M and $\mathrm{M}-\mathrm{C}$ ) and of the real $\mathrm{HB}$ images $(\mathrm{C}-\mathrm{M}$ and $\mathrm{M}-\mathrm{C})$, separately for each of the two conditions ( $\mathrm{O}$ and $\mathrm{AJ})$. Finally, differential effects were also observed across stimulus-categories, contrasting the effects evoked by sculpture images vs. real HB images (and vice versa) within each experimental condition. For each participant, this led to the creation of 11 contrast-images in Experiment 1 , that is one for each of the sub-conditions $(2 \times 2$ : stimulustype $\times$ stimulus-category) for each experimental condition $(\mathrm{O}$ and AJ) plus three common to all conditions (instruction, blank inter$\mathrm{val}$, and motor response); and of 10 contrast-images in Experiment 2 , that is one of each of the sub-conditions $(2 \times 2$ : stimulustype $\times$ stimulus-category) plus two common to all conditions (blank interval and motor response - see below).

These contrast-images then underwent the second step where the regressors of interest were modeled into Flexible Factorial analyses. The models considered the pattern of activation of the two stimulus-types $(\mathrm{C}$ and $\mathrm{M})$ vs. implicit baseline for each of the two stimulus-categories ( $\mathrm{S}$ and $\mathrm{HB}$ ) for each condition $(\mathrm{O}$ and $\mathrm{AJ})$ Linear contrasts were used to compare these effects. Correction

\footnotetext{
${ }^{4}$ http://www.fil.ion.ucl.ac.uk/spm

${ }^{5}$ http://Loni.ucla.edu/ICBM/ICBM_TissueProb.html
} 
for non-sphericity (Friston et al., 2002) was used to account for possible differences in error variance across conditions and any non-independent error terms for the repeated measures.

Within the Flexible Factorial analyses, the following contrasts were tested. First, the "common effects of stimulus-category" ( $\mathrm{S}$, $\mathrm{C}+\mathrm{M}$ vs. baseline) and ( $\mathrm{HB}, \mathrm{C}+\mathrm{M}$ vs. baseline) averaging across the two experimental conditions ( $\mathrm{O}$ and $\mathrm{AJ})$. Second, contrasts explored main and simple effects of stimulus-category comparing activations in response to canonical sculpture vs. canonical real human stimuli canonical sculptures (SC) vs. canonical human bodies (HBC) and vice versa across and within the two experimental conditions $(\mathrm{O}, \mathrm{AJ})$. Finally, every stimulus-type (canonical vs. modified) specific effect was assessed within stimulus-category ("S": C vs. M, M vs. C; "HB”: C vs. M, M vs. C) separately for each condition $(\mathrm{O}, \mathrm{AJ})$.

In order to analyze only activations above baseline, all contrast analyzes (in both Experiment 1 and 2) were masked inclusively for the effect under investigation (e.g., for the contrast SC-HBC during $\mathrm{AJ}$, the contrast was masked inclusively by SC_AJ). Results were thresholded at $P<0.05$ FWE corrected at the cluster or voxel level (cluster size estimated with a voxel level threshold of $P$-uncorrected $=0.001)$.

The location of the activation foci was determined in the stereotaxic space of MNI coordinates system. Those cerebral regions for which maps are provided were also localized with reference to cytoarchitectonical probabilistic maps of the human brain, using the SPM-Anatomy toolbox v1.7 (Eickhoff et al., 2005).

\section{RESULTS}

\section{PRELIMINARY BEHAVIORAL RESULTS \\ Proportion judgment}

In the preliminary behavioral study, aimed at stimulus selection, we assessed participants' capacity to recognize proportion modifications in both sculpture and real-body images. Proportion rating was taken on a dichotomous measure $(0=$ disproportioned; $1=$ proportioned). Non-parametric data analyses for related samples were carried out on the sum of the scores obtained within each stimulus classification [SC, modified sculptures (SM); HBC, modified human bodies (HBM)] testing probability rating between pairs of stimulus-combinations.

Wilcoxon signed-rank test compared, separately, scores obtained for the canonical SC images with their corresponding modified versions (SM) and scores obtained for canonical $\mathrm{HB}$ (HBC) images with their modified versions (HBM). Results revealed that the probability to rate a canonical image proportioned was greater than for the modified images in both stimulus-categories [SC-SM $=19$ positive differences, two negative differences $(N=22) ; z=3.13, p=0.002$; HBC-HBM $=19$ positive differences, two negative differences $(N=22) ; z=3.49$, $p<0.001]$. Additionally, analyses were carried out comparing proportion scores across categories (SC vs. HBC; SM vs. HBM). Results revealed that the probability to rate canonical sculpture images proportion did not differ from that of rating proportion of canonical real-body images [SC-HBC $=10$ positive differences, 11 negative differences $(N=22) ; z=0.001, p=1]$. Similarly, comparison between the modified versions of the stimuli across category showed no significant differences in proportion ascription
$[\mathrm{SM}-\mathrm{HBM}=11$ positive differences, eight negative differences $(N=22) ; z=0.46, p=0.65]$.

\section{Esthetic judgment}

Esthetic ratings were provided on a scale ranging from 0 (ugly) to 7 (beautiful). Data analysis was carried out using repeated measures general linear models (GLM) and Greenhouse-Geisser values are reported when the sphericity assumption was violated (Mauchly's Test of Sphericity, $p<0.05$ ).

To test for differences between canonical and modified stimuli across categories, a $2 \times 2$ repeated measures analysis was performed with two levels of stimulus-category ( $\mathrm{S}$ vs. HB) and two levels of stimulus-type (C vs. $\mathrm{M})$. The results revealed a main effect of stimulus-category $[\mathrm{S}>\mathrm{HB} \quad F(1,21)=9.33$, $p=0.006$, partial $\eta^{2}=0.32$, power $\left.=0.83\right]$ as well as a main effect of stimulus type $[\mathrm{C}>\mathrm{M}$; $\mathrm{SC}$ mean $=4.1, \mathrm{SD}=1.22$; $\mathrm{SM}$ mean $=3.8, \mathrm{SD}=1.29 ; \mathrm{HBC}$ mean $=3.5, \mathrm{SD}=1.1 ; \mathrm{HBM}$ mean $=3.2, \mathrm{SD}=0.9 ; F(1,21)=24, p=0.0001$, partial $\eta^{2}=0.52$, power $=0.99$ ]. These results showed that sculpture images were rated esthetically higher than real-body images and that canonical stimuli were rated higher than their corresponding modified versions in both stimulus-categories.

\section{Dynamic vs. static}

Canonical sculpture and real-body images were classified into dynamic and static according to the criteria described in the Section "Materials and Methods." Expressed dynamism was assessed by nine independent judges (inter-rater correlation coefficient $($ ICC $)=0.85 ; p<0.001)$.

To test for differences in esthetic rating between dynamic and static stimuli across categories, a $2 \times 2$ repeated measures analysis was performed with two levels of stimulus-category ( $\mathrm{S}$ vs. HB) and two levels of stimulus-dynamism (Static vs. Dynamic). The results revealed no effects of either stimulus-category or stimulus-dynamism $(p>0.05)$.

\section{fMRI behavioral results}

Behavioral data analysis was carried out on the basis of participants' responses during AJ scanning sessions. Responses were dichotomous (see Materials and Methods). Since each stimulus was repeated twice, only responses that were consistent between repetitions were used for analysis. Overall, most of the responses were congruent between repetitions (\% of congruence Experiment $1: \mathrm{SC}=95, \mathrm{SM}=93, \mathrm{HBC}=92, \mathrm{HBM}=92 ; \%$ of congruence Experiment 2: $\mathrm{SC}=95, \mathrm{SM}=93, \mathrm{HBC}=92, \mathrm{HBM}=92$ ).

A $2 \times 2$ repeated measures GLM analysis with two levels of stimulus-category ( $\mathrm{S}$ vs. $\mathrm{HB}$ ) and two levels of stimulus-type (C vs. M) was carried out considering the percentage of judged-asbeautiful responses ascribed to each stimulus type/category. On the whole, data obtained from the fMRI behavioral responses replicated the results described above for the preliminary study. Results from Experiment 1 showed a main effect of stimuluscategory $\left[\mathrm{S}>\mathrm{HB} ;\left(F(1,30)=4.29, p=0.047\right.\right.$, partial $\eta^{2}=0.13$, power $=0.52)]$ as well as a main effect of stimulus-type $[\mathrm{C}>\mathrm{M}$; $\left(F(1,30)=18.22, p<0.001\right.$, partial $\eta^{2}=0.39$, power $\left.\left.=0.99\right)\right]$. Results from Experiment 2, showed only a main effect of stimulustype $\left[\mathrm{C}>\mathrm{M} ;\left(F(1,17)=21.14, p<0.001\right.\right.$, partial $\eta^{2}=0.55$, 
power $=0.99)]$ and no significant difference in esthetic rating across categories $(p>0.05)$.

Finally, using the categorization of the canonical stimuli into dynamic $(n=8)$ and static $(n=8)$, we carried out a $2 \times 2$ repeated measures GLM analysis on esthetic rating ascribed as a function of stimulus-category (SC vs. HBC) and of stimulus-dynamism (dynamic vs. static). Results revealed no significant differences in either Experiment 1 and 2.

\section{fMRI RESULTS}

\section{Experiment 1}

Overall effect of viewing sculpture and real human body images. In the first fMRI analysis, we assessed, separately, the overall effect of viewing the sculpture (S) and the real HB images. In both cases, we pooled together brain activations in response to canonical $(\mathrm{C})$ and modified $(\mathrm{M})$ images across the two conditions (observation and esthetic judgment) and contrasted them with implicit baseline.

With respect to sculpture images, BOLD signal increase was found in the occipital lobe, inferior, and middle temporal lobe, IPL/intraparietal sulcus), pre-SMA, ventral premotor cortex, and in IFG. Signal increase was also observed in deep structures, including the hippocampus, amygdala, and insula. Most of the activations were bilateral, although more extensive in the right hemisphere (Figure 2A). The results are summarized in Table 1A.

With respect to viewing real $\mathrm{HB}$ images, BOLD signal increase was mostly found in the same areas that were activated when viewing sculpture images (Figure 2B; Table 1B). The main difference between the overall activations evoked by the two stimuluscategories laid in the lack of activation of the insular cortex when viewing real $\mathrm{HB}$ images (see between-category analysis below).

\section{Between-category differences}

Canonical sculpture vs. canonical real human body images. This analysis was carried out comparing activations associated with observation of canonical stimuli only. Direct comparison between sculpture and real $\mathrm{HB}$ images across experimental conditions (observation and esthetic judgment), revealed enhanced activation for canonical sculpture images in the fusiform gyrus bilaterally. Simple contrasts analyses within each experimental condition revealed additional enhanced activation of the antero-dorsal portion of the right insula during esthetic judgment condition (Figures 3A,B). These results are summarized in Table 2A.

Canonical real human body vs. canonical sculpture images. Direct comparison between canonical real $\mathrm{HB}$ and canonical sculpture images across experimental conditions (observation and esthetic judgment), revealed enhanced activation for real $\mathrm{HB}$ images in right thalamus and in right superior temporal sulcus (STS, $465010, K_{\mathrm{E}}=63, P$-uncorr $=0.008$ ). Contrast analysis of simple effects between stimulus-categories for each experimental condition separately revealed that these activations were particularly enhanced during esthetic judgment condition (Figures 4A,B; Table 2A).

\section{Between-types differences}

Canonical vs. modified sculpture images. The direct comparison of canonical vs. modified sculpture images produced no significant

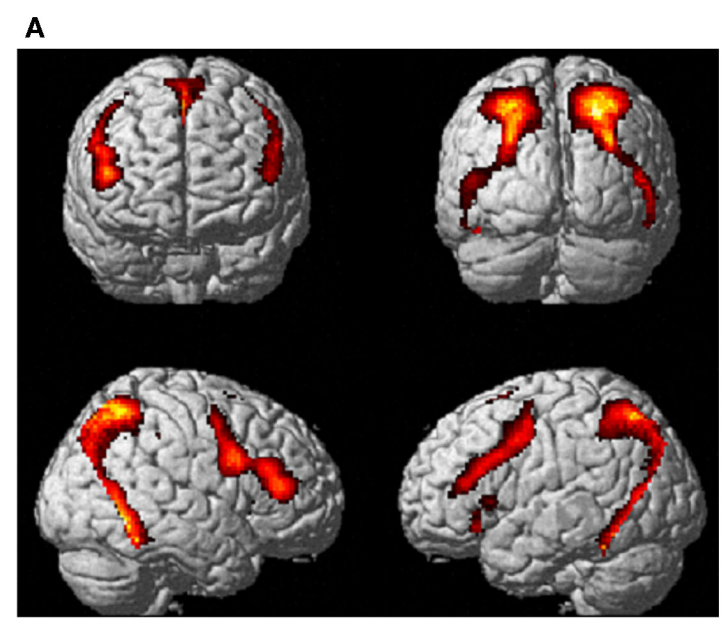

B

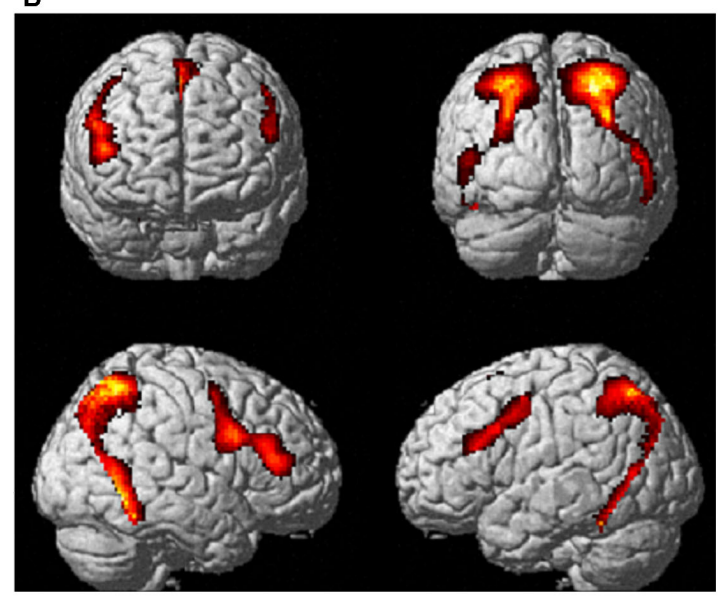

FIGURE 2 | Activations for (A) sculpture and (B) real human body images vs. implicit baseline in Experiment 1 pulling together canonical and modified stimulus-types across the two experimental conditions (observation and esthetic judgment). P-FWE corr $<0.05$.

enhanced activation for either canonical or modified images in neither of the two experimental conditions (observation and esthetic judgment). These findings are in contrast with the results obtained in our former experiment (Di Dio et al., 2007), where the direct contrast of canonical vs. modified images across all experimental conditions revealed signal increase for the canonical stimuli in some cortical areas and in right insular cortex, particularly during observation condition (see Experiment 2 below).

Canonical vs. modified real human body images. The direct comparison of canonical minus modified real HB images produced no significant enhanced activation in neither of the two experimental conditions (observation and esthetic judgment). The opposite direct comparison (modified minus canonical real $\mathrm{HB}$ images), on the other hand, produced enhanced activation for the modified images in the left amygdala during observation condition, as well as enhanced activation in a posterior cortical region straddling the inferior and middle temporal gyri during esthetic judgment condition (Table 2B). 
Table 1 | Brain activations reflecting the common effects of Canonical and Modified stimuli (pulled together) vs. baseline across conditions (observation and esthetic judgment) observed in Experiment 1 for (A) sculpture and (B) real HB images. The statistical significance refers to $P$-FWE corr $<0.05$ at the voxel level.

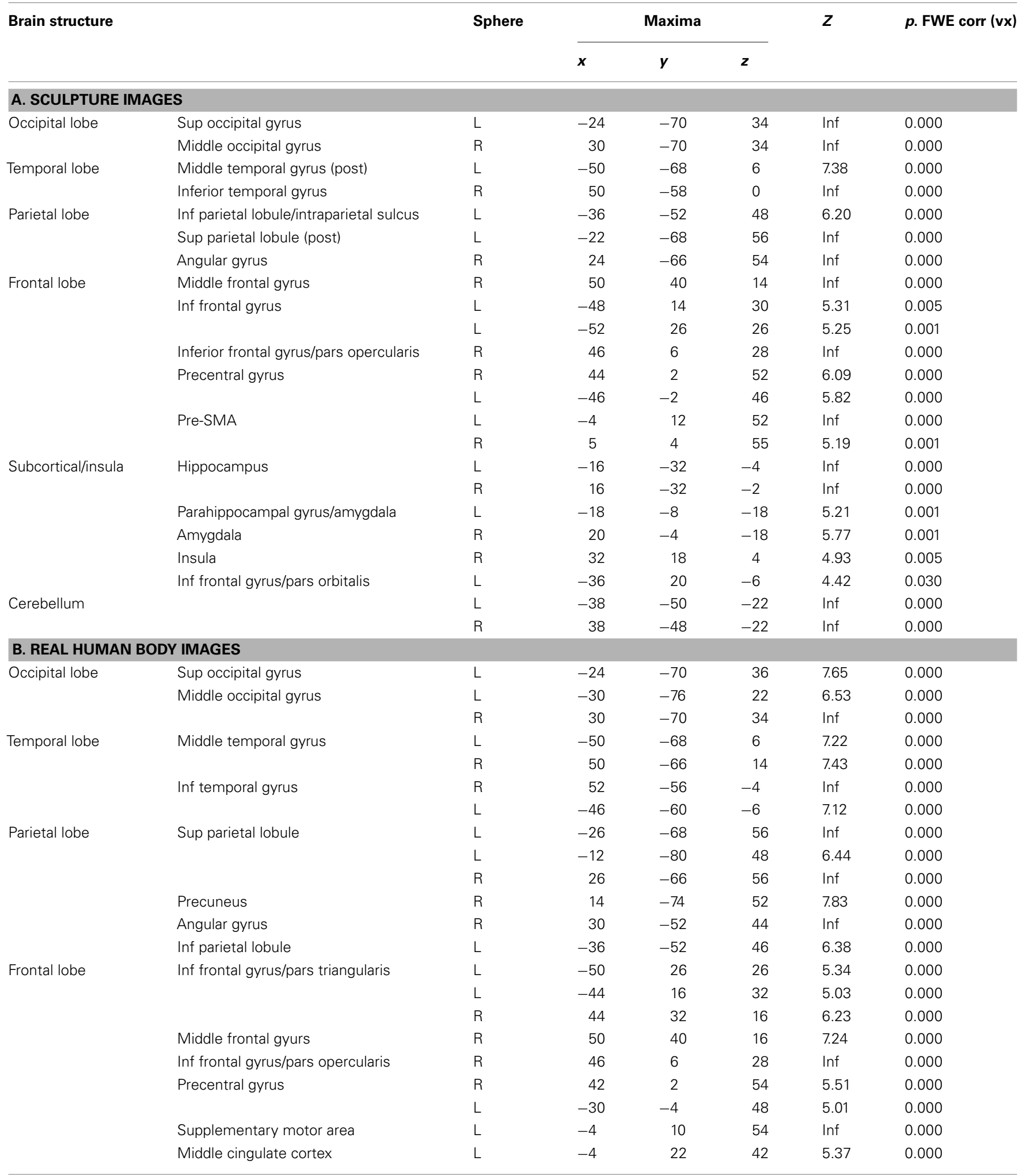


Table 1 | Continued

\begin{tabular}{|c|c|c|c|c|c|c|c|}
\hline \multirow[t]{2}{*}{ Brain structure } & & \multirow[t]{2}{*}{ Sphere } & \multicolumn{3}{|c|}{ Maxima } & \multirow[t]{2}{*}{$Z$} & \multirow[t]{2}{*}{ p. FWE corr (vx) } \\
\hline & & & $x$ & $y$ & $z$ & & \\
\hline \multirow[t]{4}{*}{ Subcortical } & Hippocampus & $L$ & -16 & -34 & -4 & $\operatorname{lnf}$ & 0.000 \\
\hline & & $L$ & -16 & -8 & -18 & 5.70 & 0.000 \\
\hline & & $\mathrm{R}$ & 18 & -32 & -0 & $\operatorname{lnf}$ & 0.000 \\
\hline & Amygdala & $\mathrm{R}$ & 18 & -6 & -16 & 7.02 & 0.000 \\
\hline \multirow[t]{2}{*}{ Cerebellum } & & $L$ & -38 & -50 & -22 & $\operatorname{lnf}$ & 0.000 \\
\hline & & $\mathrm{R}$ & 38 & -48 & -22 & $\operatorname{lnf}$ & 0.000 \\
\hline
\end{tabular}

A
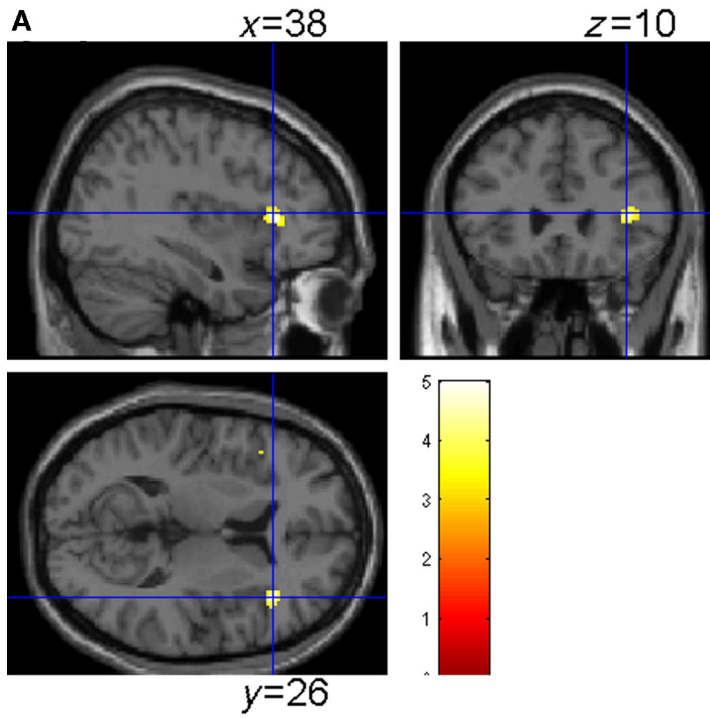

B

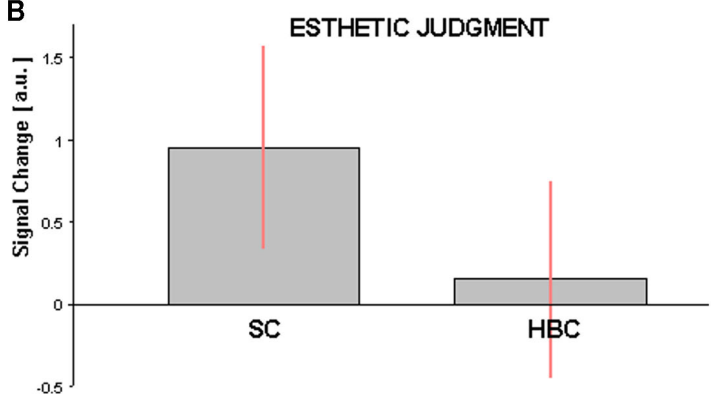

FIGURE 3 | (A) Activation in the contrast canonical sculpture minus canonical real human body images (masked incl. by canonical sculpture images) during esthetic judgment condition in Experiment 1. (B) Activity profile within right insula (38 2610 ) in arbitrary units (a.u.), $\pm 10 \%$ confidence intervals ( $P$-FWE corr $<0.05$ ).

\section{EXPERIMENT 2}

\section{fMRI results}

Overall effect of viewing sculpture and real human body images. In this analysis, we assessed, separately, the overall effect of viewing both sculpture $(\mathrm{S})$ and real HB images pooling together brain activations in response to canonical $(\mathrm{C})$ and modified $(\mathrm{M})$ images across the two conditions (observation and esthetic judgment) with respect to implicit baseline.

Figure 5A shows BOLD signal increase for sculpture images. Most of the activations replicated those observed in Experiment 1 (see Table 3A). Activated areas included occipital cortex, fusiform gyrus, lingual gyrus, posterior parietal cortex, IPL, pre-SMA, premotor cortex, and IFG. Additionally, enhanced activations were observed in deep structures, including hippocampus, amygdala, and the anterior insula. Most of the activations were bilateral. Finally, differently from Experiment 1, signal increase was also found in medial frontal areas, including right anterior cingulate cortex and left orbitofrontal cortex.

Figure 5B shows activations relative to viewing real $\mathrm{HB}$ images. Similarly to Experiment 1, BOLD signal increase was found in the same areas that were activated when viewing sculpture images, the major difference being an additional activation at the level of the basal ganglia nuclear complex (Table 3B).

\section{Between-category differences}

Canonical sculpture vs. canonical real human body images. Direct comparison between canonical sculpture and canonical real HB images across experimental conditions (observation and esthetic judgment) revealed greater activations for sculpture images in lingual and fusiform gyri. Additional activations were observed from simple contrast analyses. More specifically, during observation condition there was increased activation for canonical sculpture vs. canonical HB images in right cuneus, right IPL, right IFG pars triangularis, and pars opercularis, and in the anterior dorsal part of right insula (Table 4A).

Canonical real human body vs. canonical sculpture images. The direct comparison between canonical real HB vs. canonical sculpture across experimental conditions (observation and esthetic judgment) revealed enhanced activations bilaterally in the caudal part of the temporal lobe straddling the middle and superior temporal gyri and extending medially to include the STS. Simple contrast analyzes showed that activation of left STS was particularly strong during esthetic judgment condition (Table 4A).

\section{Between-type differences}

Canonical vs. modified sculpture images. The direct comparison of canonical vs. modified sculpture images revealed significant differences during observation condition only. More specifically, signal increase was observed for canonical images in the caudal 
Table 2 | Brain activity reflecting the effect of stimulus (A) category (canonical sculpture and canonical real HB images) and (B) type (canonical and modified) for the two conditions $(O=$ observation; $A J=$ esthetic judgment) in Experiment 1; $S C=$ sculpture canonical; $S M=s c u l p t u r e$ modified; $\mathbf{H B C}=$ real human body canonical; $\mathbf{H B M}=$ real human body modified. The reported statistical significance is at cluster level and refers to activations significant $(P$-FWE corr $<0.05)$ at the cluster and/or voxel level.

\begin{tabular}{|c|c|c|c|c|c|c|c|c|c|}
\hline \multirow[t]{2}{*}{ Contrast } & \multirow[t]{2}{*}{ Condition } & \multirow[t]{2}{*}{ Brain structure } & \multirow[t]{2}{*}{$K_{\mathrm{E}}$} & \multirow[t]{2}{*}{ p. FWE corr cluster level } & \multirow[t]{2}{*}{$Z$} & \multirow[t]{2}{*}{ Sphere } & \multicolumn{3}{|c|}{ Maxima } \\
\hline & & & & & & & $x$ & $y$ & $z$ \\
\hline \multirow[t]{8}{*}{$\mathrm{SC}-\mathrm{HBC}$} & $\mathrm{O}+\mathrm{AJ}$ & Fusiform gyrus & 292 & 0.000 & 5.26 & $\mathrm{~L}$ & -30 & -36 & -18 \\
\hline & & & & & 4.49 & $\mathrm{~L}$ & -26 & -56 & -14 \\
\hline & & & 190 & 0.001 & 4.63 & $\mathrm{R}$ & 34 & -46 & -10 \\
\hline & $\mathrm{O}$ & Fusiform gyrus & 502 & 0.000 & 5.55 & $\mathrm{R}$ & 30 & -46 & -14 \\
\hline & & & & & 4.10 & $\mathrm{R}$ & 28 & -56 & -10 \\
\hline & & & 390 & 0.000 & 5.21 & $\mathrm{~L}$ & -26 & -42 & -16 \\
\hline & & & & & 5.05 & $L$ & -32 & -38 & -20 \\
\hline & AJ & Insula & 77 & 0.074 & 4.99 & $\mathrm{R}$ & 38 & 26 & 10 \\
\hline $\mathrm{HBC}-\mathrm{SC}$ & $\mathrm{O}+\mathrm{AJ}$ & Thalamus & 134 & 0.006 & 4.42 & $\mathrm{R}$ & 2 & -12 & 14 \\
\hline \multirow[t]{2}{*}{ HBM-HBC } & $\mathrm{O}$ & Amygdala & 97 & 0.030 & 5.13 & $L$ & -30 & -4 & -14 \\
\hline & & Inf temporal gyrus (post) & 46 & 0.321 & 5.02 & $\mathrm{~L}$ & -48 & -42 & -16 \\
\hline $\mathrm{HBM}-\mathrm{HBC}$ & AJ & Inf temporal gyrus (post) & 220 & 0.000 & 4.83 & $\mathrm{R}$ & 50 & -54 & -6 \\
\hline
\end{tabular}

part of right middle temporal gyrus, IFG pars triangularis, and, crucially, in right anterior dorsal insular cortex (Figures 6A,B; Table 4B).

The contrast modified vs. canonical sculpture images revealed signal increase during esthetic judgment condition in right supramarginal gyrus and right ventral premotor cortex (BA44; Table 4B).

\section{Canonical vs. modified human body images}

The comparison of canonical vs. modified $\mathrm{HB}$ images revealed no significant differences. The opposite contrast (modified vs. canonical HB images), on the other hand, revealed signal increase for the modified images in ventral premotor cortex (BA44) during observation condition and in superior parietal lobule, inferior temporal gyrus, and fusiform gyrus during esthetic judgment condition. All activations were lateralized in the right hemisphere (Table 4B).

\section{DISCUSSION}

In a previous study we showed that activation of the anterior sector of the right insula is associated with the hedonic state underpinning esthetic experience during the observation of artworks (Di Dio et al., 2007). The main aim of the present study was to investigate whether this specific hedonic response is also present during the observation of non-art biological stimuli. For this purpose, we compared brain activations when participants observed sculpture images with brain activations during the observation of real $\mathrm{HB}$ represented by photographs of young athletes.

The global pattern of cortical activations during the presentation of sculptures and real $\mathrm{HB}$ was very similar. Activations included visual occipital and temporal areas, IPL/intraparietal sulcus, ventral premotor cortex, and IFG. Signal increase was also observed in deep structures, such as the hippocampus and amygdala. Most of the activations were bilateral, although more extensive in the right hemisphere. The direct comparison between SC and canonical real bodies highlighted, however, some important differences. The observation of sculpture images determined, relative to real $\mathrm{HB}$ images, a greater activation of right anterior dorsal insula, as well as activation of some visual areas and, in particular, of fusiform gyrus. The opposite contrast (HB minus sculpture images) showed a greater activation of the STS.

It is known from both monkey (see Desimone et al., 1984; Tsao et al., 2006; Gross, 2008) and human studies that portions of the inferotemporal lobe and of its human homolog (the fusiform gyrus), play a crucial role in the processing of faces (for review see McKone and Kanwisher, 2005; Gross, 2008). Furthermore, it was also shown that some sectors of fusiform gyrus encode, with nearly the same level of selectivity, images of human body (Peelen and Downing, 2004; Schwarzlose et al., 2005). In this light, it is plausible that the fusiform activation observed in the present study reflected a detailed visual analysis of the physical aspects of the body (e.g., size, shape, proportion) of the sculpture as compared to real HB images.

The comparison between real HB vs. sculpture images showed a consistent activation of the STS. STS is a region known to be involved in visual processing of movement of body parts. Thus, STS activation was likely due to a matching between the observed $\mathrm{HB}$ images and the representation of body movement encoded in this region (see Perrett et al., 1989; Allison et al., 2000; Pelphrey et al., 2004; Thompson et al., 2005). Note that, although in the 


\section{A}
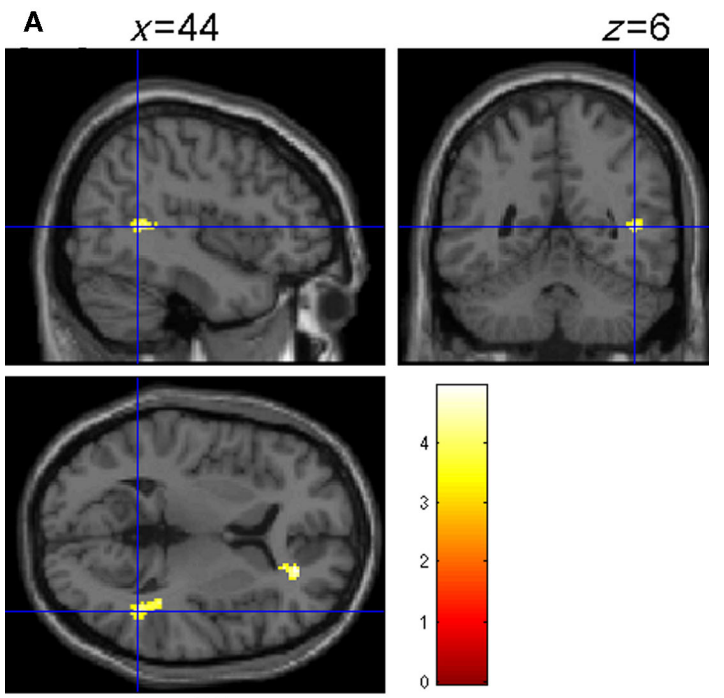

$y=-50$

B

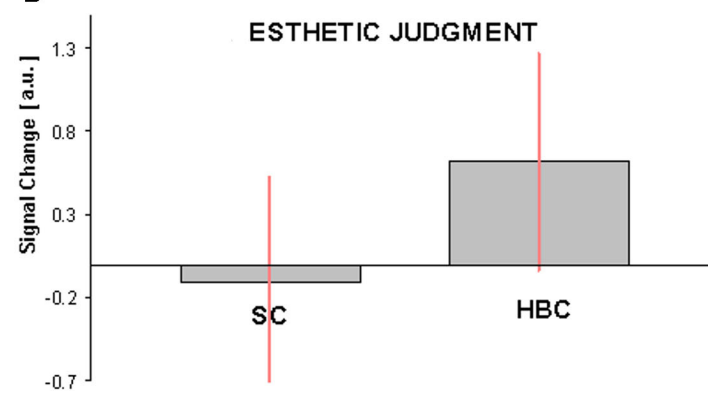

FIGURE 4 | (A) Activation in the contrast canonical real human body minus canonical sculpture images (masked incl. by canonical human body images) during esthetic judgment condition in Experiment 1. (B) Activity profile within right STS (44 506 ) in arbitrary units (a.u.), $\pm 10 \%$ confidence intervals ( $P$-FWE corr $<0.05$ ).

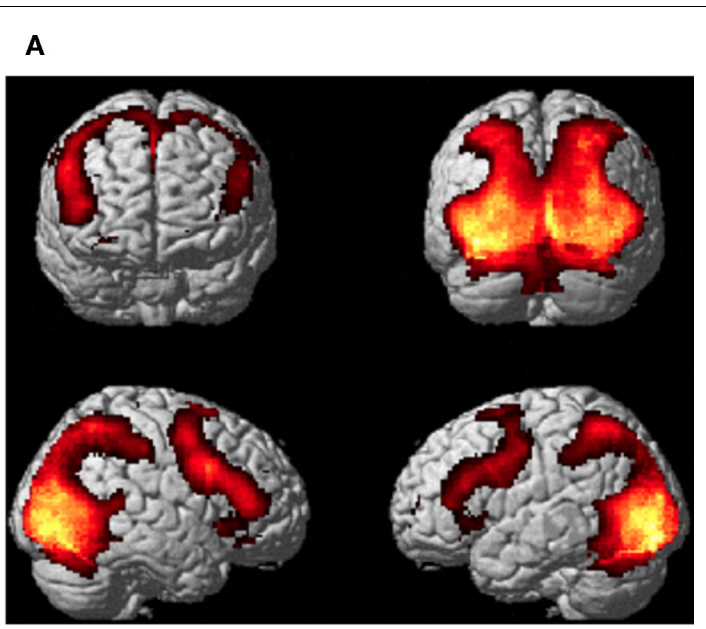

B

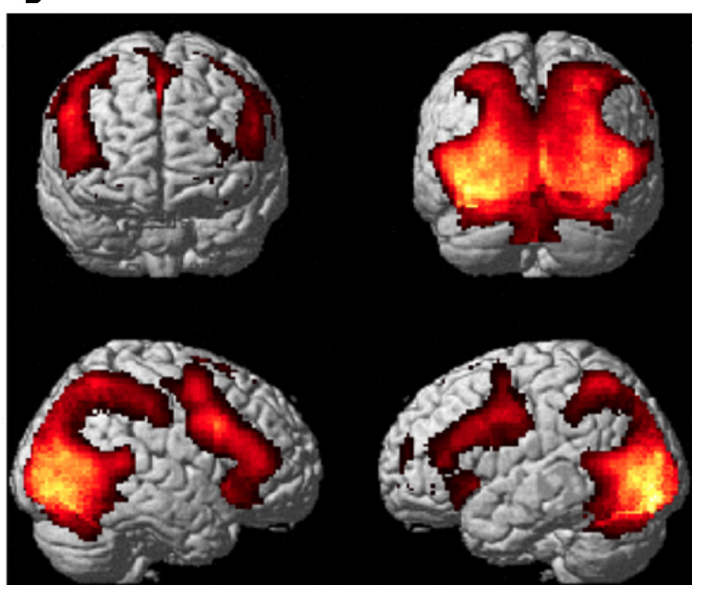

FIGURE 5 | Activations for (A) sculpture and (B) real human body images vs. implicit baseline in Experiment 2 pulling together canonical and modified stimulus-types across the two experimental conditions (observation and esthetic judgment). P-FWE corr $<0.05$. present study we used static stimuli, there is evidence that these stimuli, when implying motion, are able to activate visual areas encoding overt movements, as shown for area MT/V5 by Kourtzi and Kanwisher (2000).

In the present study, both sculpture and real-body images contained an even number of static and dynamic stimuli. It is then likely that activation differences observed between real-body and sculpture images were not be ascribed to differences in some stimulus properties (such as dynamism - see also behavioral results), but rather to different attention deployment in the two cases. Attention was more focused on action in the case of real human images, whilst it was more focused on the physical aspects of the body in the case of sculpture images. In turn, these different attention allocations could be related to different attitudes toward the presented images. In the case of the real HB, the implicit attitude of the observers would be that of trying to understand the meaning of the represented gestures and, possibly, the intention of the observed individuals. In contrast, the sculptures constitute an artistic representation of the $\mathrm{HB}$ and the spontaneous attitude of the observers would be that of exploring them with the purpose of appreciating their physical properties.

The most important finding of our study lays, however, in the activation of right insula in the contrast sculpture vs. real HB images. The activated part of the insula was located in its rostrodorsal sector. This sector corresponds to the insular region also found activated in our previous study in the contrast canonical vs. modified sculpture images (Di Dio et al., 2007) and confirmed from the same contrast in Experiment 2 of the present study. Since canonical proportions are positively related to esthetic evaluation of sculpture images, we interpreted this activation as the hedonic signature of esthetic experience when viewing artworks.

Insula is an extremely complex and heterogeneous structure including a posterior granular (sensory part), a central large dysgranular, and a small rostro-ventral agranular (motor and vegetative parts) sector (see Mesulam and Mufson, 1982, 1985; Augustine, 1996). A recent meta-analysis of the human insula by 
Table 3 | Brain activations reflecting the common effects of canonical and Modified stimuli (pulled together) vs. baseline across conditions (observation and esthetic judgment) observed in Experiment $\mathbf{2}$ for (A) sculpture and (B) real HB images. The statistical significance refers to $P$-FWE corr $<0.05$ at the voxel level.

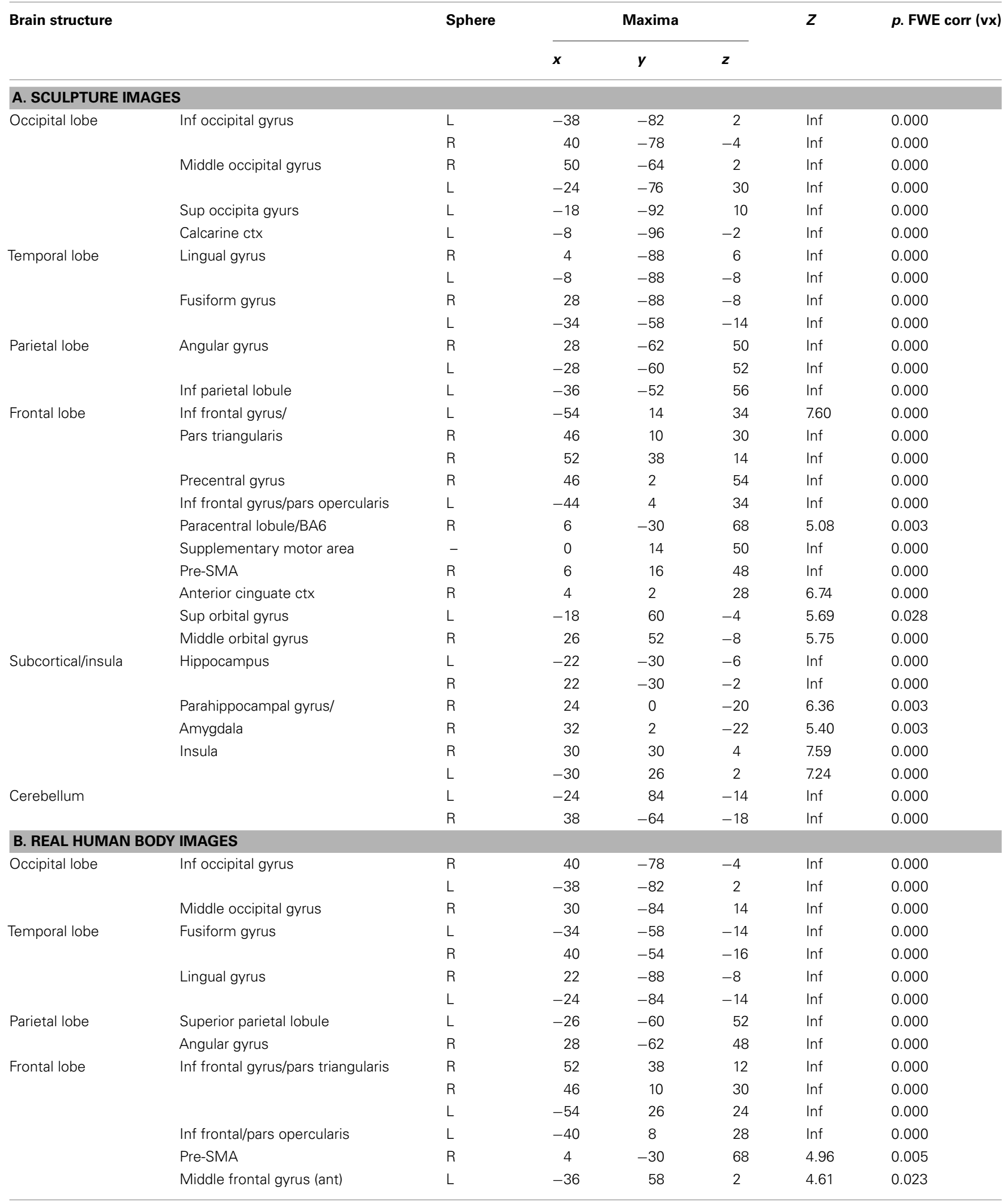


Table 3 | Continued

\begin{tabular}{|c|c|c|c|c|c|c|c|}
\hline Brain structure & & Sphere & \multicolumn{3}{|c|}{ Maxima } & $z$ & p. FWE corr (vx) \\
\hline & Middle orbital gyrus & $\mathrm{L}$ & -28 & 52 & -6 & 4.45 & 0.043 \\
\hline \multirow[t]{5}{*}{ Subcortical } & Hippocampus & $L$ & -22 & -30 & -6 & $\operatorname{lnf}$ & 0.000 \\
\hline & & $\mathrm{R}$ & 22 & -30 & -2 & $\operatorname{lnf}$ & 0.000 \\
\hline & Amygdala & $\mathrm{L}$ & -20 & -6 & -12 & 4.69 & 0.011 \\
\hline & Inf frontal gyrus/pars orbitalis & $\mathrm{R}$ & 34 & 26 & -2 & 8.60 & 0.000 \\
\hline & Insula & $\mathrm{L}$ & -30 & 26 & 2 & 7.90 & 0.000 \\
\hline Basal ganglia & Globus pallidus & $\mathrm{L}$ & -12 & -2 & -2 & 5.34 & 0.001 \\
\hline
\end{tabular}

Table 4 | Brain activity reflecting the effect of stimulus (A) Category (canonical sculpture and canonical real HB images) and (B) Type (canonical and modified) for the two conditions ( $O=$ observation; $A J=$ esthetic judgment) in Experiment 2; $\mathbf{S C}=\mathbf{s c u l p t u r e}$ canonical; $\mathbf{S M}=\mathbf{S c u l p t u r e}$ modified; $\mathbf{H B C}=$ real human body canonical; $\mathbf{H B M}=$ real human body modified. The reported statistical significance is at cluster level and refers to activations significant $(P$-FWE corr $<0.05)$ at the cluster and/or voxel level.

\begin{tabular}{|c|c|c|c|c|c|c|c|c|c|}
\hline Contrast & Condition & Brain structure & $\kappa_{\mathrm{E}}$ & $\begin{array}{l}\text { p. FWE corr } \\
\text { cluster level }\end{array}$ & $Z$ & Sphere & \multicolumn{3}{|c|}{ Maxima } \\
\hline \multirow[t]{11}{*}{$\mathrm{SC}-\mathrm{HBC}$} & $\mathrm{O}+\mathrm{AJ}$ & Lingual gyrus & 1011 & 0.000 & 5.86 & $\mathrm{R}$ & 10 & -84 & -4 \\
\hline & & Fusiform gyrus & 160 & 0.095 & 4.67 & $\mathrm{R}$ & 30 & -42 & -20 \\
\hline & $\mathrm{O}$ & Lingual gyrus & 7565 & 0.000 & 5.87 & $\mathrm{R}$ & 16 & -84 & -6 \\
\hline & & Fusiform gyrus & & & 5.23 & $\mathrm{~L}$ & -36 & -44 & -10 \\
\hline & & & & & 5.02 & $\mathrm{R}$ & 30 & -38 & -20 \\
\hline & & Cuneus & & & 5.12 & $\mathrm{R}$ & 22 & -66 & 28 \\
\hline & & & & & 4.58 & $\mathrm{R}$ & 26 & -66 & 50 \\
\hline & & Insula & 134 & 0.180 & 5.21 & $\mathrm{R}$ & 30 & 26 & 6 \\
\hline & & Inf parietal lobule & 263 & 0.025 & 4.94 & $\mathrm{R}$ & 50 & -34 & 42 \\
\hline & & Fusiform gyrus & & & 4.45 & $\mathrm{R}$ & 30 & -40 & -22 \\
\hline & & Lingual gyrus & & & 4.36 & $\mathrm{R}$ & 16 & -84 & -8 \\
\hline \multirow[t]{7}{*}{$\mathrm{HBC}-\mathrm{SC}$} & $\mathrm{O}+\mathrm{AJ}$ & Sup/mid temporal gyrus & 297 & 0.017 & 4.79 & $\mathrm{R}$ & 64 & -40 & 14 \\
\hline & & & & & 4.05 & $\mathrm{R}$ & 54 & -52 & 16 \\
\hline & & & & & 3.44 & $\mathrm{R}$ & 58 & -38 & 6 \\
\hline & & Sup/mid temporal gyrus & 429 & 0.004 & 4.79 & $\mathrm{~L}$ & -48 & -64 & 18 \\
\hline & & & & & 4.57 & $\mathrm{~L}$ & -52 & -54 & 12 \\
\hline & AJ & Sup/mid temporal gyrus & 228 & 0.054 & 3.98 & $\mathrm{~L}$ & -54 & -52 & 12 \\
\hline & & & & & 3.54 & $\mathrm{~L}$ & -46 & -54 & 6 \\
\hline \multicolumn{10}{|c|}{ B. EFFECTS OFTYPE } \\
\hline SC-SM & $\mathrm{O}$ & Inf frontal gyrus/pars triangularis & 253 & 0.051 & 6.47 & $\mathrm{R}$ & 38 & 30 & 0 \\
\hline HBM-HBC & & Sup parietal lobule & 306 & 0.038 & 4.11 & $\mathrm{R}$ & 30 & -64 & 30 \\
\hline
\end{tabular}



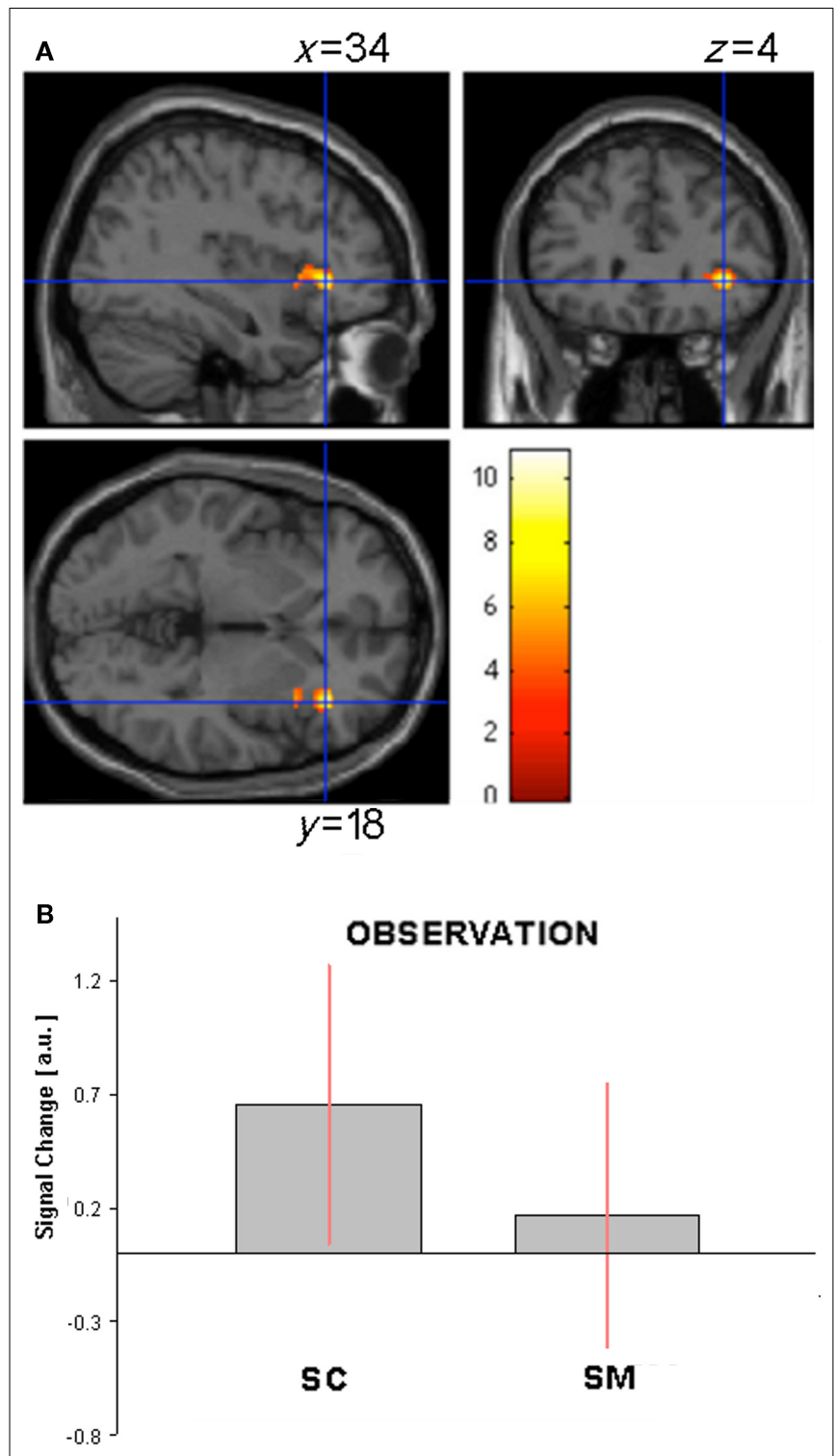

FIGURE 6 | (A) Activation in the contrast canonical minus modified sculpture images (masked incl. by canonical sculpture images) during observation condition in Experiment 2. (B) Activity profile within right anterior insula (34 18 4) in arbitrary units (a.u.), $\pm 10 \%$ confidence intervals (P-FWE corr $<0.05)$.

Kurth et al. (2010) revealed four functional distinct regions corresponding to sensory-motor, olfacto-gustatory, social-emotional, and cognitive networks of the brain. Social-emotional aspects activate the ventro-rostral part of the insula while all tested functions, except for sensory-motor function, overlap on its anterior dorsal portion. These data allow one to specify better the functional role of this region in mediating hedonic experiences when viewing artworks. This region is not encoding the mere emotional aspect of the stimuli, but integrates cognitive and emotional processes to create a coherent experience of the attended stimuli. Although activation of this region is not uniquely deputed to esthetic experience (see Kurth et al., 2010), our results indicate that it plays a fundamental role in providing an hedonic quality to art processing.

One may argue that insular activation observed for sculpture images, and not for real HB stimuli, could have been triggered by the sculptures complete nudity, a factor that was not counterbalanced between categories. In this respect, some experimental evidence coming from studies investigating the neural correlates of emotional response to arousing stimuli report insula activation. Often, the arousing stimuli represent film clips or photographs depicting nudes and sex scenes (e.g., Stoléru et al., 1999; Gizewski et al., 2006; Safron et al., 2007). In these studies, right ventral insula and/or left insula were found activated when attending arousing stimuli. The rostro-ventral insular sector found activated in these studies is different from the more dorsal sector observed in our study. Anterior ventral insula is often associated with a representation of autonomic states (e.g., Critchley et al., 2002) and with the presentation of stimuli holding a socio-emotional status (see Kurth et al., 2010 for a review). Most noteworthy, our results indicate that the insular sector found activated in the contrast sculpture vs. real HB stimuli showed also a lower activation in association with decreased esthetic valence conveyed by the proportion-modified stimuli. For this reason, we suggest that the right antero-dorsal insular activation observed for sculpture images in the present study is evoked by an hedonic state associated with the esthetic dimension of the sculptures.

Insular activation was absent in the case of observation of real HB images, irrespective of proportion modification. It is worth noting, in this respect, that behavioral data showed that proportion affected esthetic rating in both stimulus-categories; namely, the canonical images were preferred to modified images also in the case of real HB. What these data seem to suggest is that the enhanced insular activation observed for sculpture images compared to real-body images, and particularly for canonical ones, emerged from attendance to specific physical properties of the sculpture images that, when altered, determined a diminished hedonic response in the viewer. This specific hedonic response was not present when judging the esthetics of real-body images. This does not imply that there is no esthetic experience associated with the viewing of real-body images. However, our data show that this experience does not have the same neural substrates as those underpinning the viewing of sculptures. Exploration of the neural correlates associated with esthetic experience for real $\mathrm{HB}$ was beyond the purpose of the present study and we cannot assert any conclusions on this issue.

\section{CONCLUSION}

Here we tested whether the neural activations underpinning hedonic experience when viewing an artistic representation of the $\mathrm{HB}$ (masterpieces of classical art) are also present when observing images of non-art biological stimuli (real HB). Imaging results indicated that esthetic experience for artworks recruited the anterior sector of right dorsal insula. This sector was not activated when attending real HB images. This indicates that esthetic experience for artworks and non-art biological stimuli does not share the same neural substrate.

It would be too reductive, however, to think that esthetic experience occurs because of the activation of the antero-dorsal insula 
alone. Our view is that esthetic experience derives from a joint activity of neural cortical populations responsive to specific elementary or high order features present in works of art and neurons located in emotion controlling centers. A recent meta-analysis on the functional properties of the different sectors of the insula indicates that the insular region we found activated during the viewing of artworks does not merely mediate emotions but links emotion

\section{REFERENCES}

Allison, T., Puce, A., and McCarthy, G. (2000). Social perception from visual cues: role of the STS region. Trends Cogn. Sci. 4, 267-278.

Andersson, J. L., Hutton, C., Ashburner, J., Turner, R., and Friston, K. (2001). Modeling geometric deformations in EPI time series. Neuroimage 13, 903-919.

Augustine, J. R. (1996). Circuitry and functional aspect of the insular lobe in primates including humans. Brain Res. Rev. 22, 229-244.

Cela-Conde, C. J., Ayala, F. J., Munar, E., Maestu, F., Nadal, M., Capo, M. A., del Rio, D., Lopez-Ibor, J. J., Ortiz, T., Mirasso, C., and Marty, G. (2009). Sex-related similarities and differences in the neural correlates of beauty. Proc. Natl. Acad. Sci. 106, 3847-3852.

Craig, A. D. (2003). Interoception: the sense of the physiological condition of the body. Curr. Opin. Neurobiol. $13,500-505$.

Critchley, H. D., Melmed, R. N., Featherston, E., Mathias, C. J., and Dolan, R. J. (2002). volitional control of autonomic arousal: a functional magnetic resonance study. Neuroimage 16, 909-919.

Critchley, H. D., Rotshtein, P., Nagai, Y., O'Doherty, J., Mathias, C. J., and Dolan, R. J. (2005). Activity in the human brain predicting differential heart rate responses to emotional facial expressions. Neuroimage 24, 751-762.

Critchley, H. D., Wiens, S., Rotshtein, P., Ohman, A., and Dolan, R. J. (2004). Neural systems supporting interoceptive awareness. Nat. Neurosci. 7, 189-195.

Cupchik, G. C., Vartanian, O., Crawley, A., and Mikulis, D. J. (2009). Viewing artworks: contributions of cognitive control and perceptual facilitation to aesthetic experience. Brain Cogn. 70, 84-91.

Dale, A. M. (1999). Optimal experimental design for event-related fMRI. Hum. Brain Mapp. 8, 109-114.

Damasio, A. (1999). The Feeling of What Happens: Body and Emotion in the Making of Consciousness. New York: Harcourt Brace.

Damasio, A. R., Grabowski, T. J., Bechara, A., Damasio, H., Ponto,
L. L., Parvizi, J., and Hichwa, R. D. (2000). Subcortical and cortical brain activity during the feelNeurosci. 3, 1049-1056.

Desimone, R., Albright, T. D., Gross, C. G., and Bruce, C. (1984). Stimulusselective properties of inferior temNeurosci. 4, 2051-2062.

Di Dio, C., and Gallese, V. (2009). Neuroesthetics: a review. Curr. Opin. Neurobiol. 19, 682-687.

Di Dio, C., Macaluso, E., and Rizzolatti, G. (2007). The golden beauty: brain response to classical and renaissance doi:10.1371/journal.pone.0001201

Dupont, S., Bouilleret, V., Hasboun, D., Semah, F., and Baulac, M. (2003). Functional anatomy of the insula: new insights from imaging. Surg. Radiol. Anat. 25, 113-119.

Eickhoff, S. B., Stephan, K. E., Mohlberg, H., Grefkes, C., Fink, G. R., Amunts, K., and Zilles, K. (2005). A new SPM toolbox for combining probabilistic cytoarchitectonic maps and functional imaging data. Neuroimage 25, 1325-1335.

Freedberg, D., and Gallese, V. (2007). Motion, emotion and empathy in esthetic experience. Trends Cogn. Sci. (Regul. Ed.) 11, 197-203.

Friston, K. J., Glaser, D. E., Henson, R. N., Kiebel, S., Phillips, C., and Ashburner, J. (2002). Classical and Bayesian inference in neuroimaging: applications. Neuroimage 16, 484-512.

Friston, K. J., Holmes, A. P., and Worsley, K. J. (1999). How many subjects constitute a study? Neuroimage 10 , 1-5.

Friston, K. J., Williams, S., Howard, R., Frackowiak, R. S., and Turner, R. fMRI time-series. Magn. Reson. Med. $35,346-355$.

Gizewski, E. R., Krause, E., Karama, S. Baars, A., Senf, W., and Forsting, M. (2006). There are differences in cerebral activations between females in distinct menstrual phases during viewing of erotic stimuli: a fMRI study. Exp. Brain Res. 174, 101-108.

Gross, C. G. (2008). Single neuron studies of inferior temporal cortex. Neuropsychologia 45, 841-852. ing of self-generated emotions. Nat. poral neurons in the macaque. $J$. sculptures. PLoS ONE 11, e1201. (1996). Movement-related effects in

to cognition. We suggest that this binding plays a fundamental role in determining the hedonic dimension of esthetic experience for artworks.

\section{ACKNOWLEDGMENTS}

We are grateful to Fondazione Cassa di Risparmio di Parma (CARIPARMA) for providing the facilities to conduct this study.

Jacobsen, T., Schubots, R. I., Hofel, L. and Cramon, D. V. (2006). Brain Correlates of aesthetic judgment of beauty. Neuroimage 29, 276-285.

Kawabata, H., and Zeki, S. (2004). Neural correlates of beauty. J. Neurophysiol. 91, 1699-1705.

Kourtzi, Z., and Kanwisher, N. (2000). Activation in human MT/MST by static images with implied motion. J. Cogn. Neurosci. 12, 48-55.

Kurth, F., Zilles, K., Fox, P. T., Laird, A. R., and Eickhoff, S. B. (2010). A link between the systems: functional differentiation and integration within the human insula revealed by metaanalysis. Brain Struct. Funct. 214 519-534.

Lacey, S., Hagtvedt, H., Patrick, V. M. Anderson, A., Still, R., Deshpande, G., Hu, X., Sato, J. R., Reddy, S., and Sathian, K. (2011). Art for reward's sake: visual art recruits the ventral striatum. Neuroimage 55, 420-433.

McKone, E., and Kanwisher, N. (2005). "Does the human brain process objects of expertise like faces? A review of the evidence," in From Monkey Brain to Human Brain, eds S. Dehaene, J. R. Duhamel, M. D. Hauser, and G. Rizzolatti (Cambridge, MA: MIT Press), 339-356.

Mesulam, M. M., and Mufson, E. J. (1982). Insula of the old world monkey (III): efferent cortical output and comments on function. J. Comp. Neurol. 212, 38-52.

Mesulam, M. M., and Mufson, E. J. (1985). The insula of Reil in man and monkey. Cereb. cortex 4, 179-226.

Peelen, M. V., and Downing, P. E. (2004) Selectivity for the human body in the fusiform gyrus. J. Neurophysiol. 93, 603-608.

Pelphrey, A. K., Morris, J. P., and Mccarthy, G. (2004). Grasping the intentions of others: the perceived intentionality of an action influences activity in the superior temporal sulcus during social perception. J. Cogn. Neurosci. 16, 1706-1716.

Perrett, D. I., Harries, M. H., Bevan, R. Thomas, S., Benson, P. J., Mistlin, A J., Chitty, A. J., Hietanen, J. K., and Ortega, J. E. (1989). Frameworks of analysis for the neural representation of animate objects and actions. J. Exp. Biol. 146, 87-113.

Safron, A., Barch, B., Bailey, J. M., Gitelman, D. R., Parrish, T. B., and Reber,
P. J. (2007). Neural correlates of sexual arousal in homosexual and heterosexual men. Behav. Neurosci. 121, 237-248.

Schwarzlose, R. F., Baker, C. I., and Kanwisher, N. (2005). Separate face and body selectivity on the fusiform gyrus. J. Neurosci. 25, 11055-11059.

Stoléru, S., Grégoire, M. C., Gérard, D., Dcety, J., Lafarge, E., Cinotti, L., Lavenne, F., Le Bars, D., VernetMaury, E., Rada, H., Collet, C., Mazoyer, B., Forest, M. G., Magnin, F., Spira, A., and Comar, D. (1999). Neuroanatomical correlates of visually evoked sexual arousal in human males. Arch. Sex. Behav. 28, 1-21.

Thompson, J. C., Clarke, M., Stewart, T., and Puce, A. (2005). Configural processing of biological movement in human superior temporal sulcus. J. Neurosci. 25, 9059-9066.

Tsao, D. Y., Freiwald, W. A., Tootell, R. B., and Livingstone, M. S. (2006). A cortical region consisting entirely of face-selective cells. Science 311 , 670-674.

Vartanian, O., and Goel, V. (2004). Neuroanatomical correlates of aesthetic preference for paintings. Neuroreport 15, 893-897.

Worsley, K. J., and Friston, K. J. (1995). Analysis of fMRI time-series revisited - again. Neuroimage 2, 173-181.

Conflict of Interest Statement: The authors declare that the research was conducted in the absence of any commercial or financial relationships that could be construed as a potential conflict of interest.

Received: 28 March 2011; accepted: 31 October 2011; published online: 18 November 2011.

Citation: Di Dio C, Canessa N, Cappa SF and Rizzolatti $G$ (2011) Specificity of esthetic experience for artworks: an fMRI study. Front. Hum. Neurosci. 5:139. doi 10.3389/fnhum.2011.00139

Copyright (c) 2011 Di Dio, Canessa, Cappa and Rizzolatti. This is an openaccess article subject to a non-exclusive license between the authors and Frontiers Media SA, which permits use, distribution and reproduction in other forums, provided the original authors and source are credited and other Frontiers conditions are complied with. 